\title{
Evaluation of a Heat Release Rate based on Massively Generated Simulations and Machine Learning Approach
}

\author{
Mateusz Fliszkiewicz*, Adam Krasuski* and Karol Krenski* \\ ${ }^{*}$ Section of Computer Science, \\ The Main School of Fire Service \\ Słowackiego 52/54, \\ 01-629 Warsaw, Poland \\ fliszkiewicz@inf.sgsp.edu.pl, krasuski@inf.sgsp.edu.pl, krenski@inf.sgsp.edu.pl
}

\begin{abstract}
We present an approach for evaluation of a heat release rate of compartment fires. The approach is based on the idea of matching the actual condition of the fire to the pregenerated CFD simulations. We use an IR image of imprint of the temperature on the ceiling as a similarity relationship between actual fire and the set of the simulations. We extract the invariants, features and similarity measures of the fires using machine learning approach.
\end{abstract}

Index Terms-Inverse Fire Modelling, Artificial Intelligence, Classification, Image Processing, Fire Services

\section{INTRODUCTION}

$\mathbf{M}$ ODELING of fire dynamics in buildings attracts many researchers from the fire safety science domain [1], [2]. The achievements of the research are successfully implemented in the processes of building design such as installation of fire detection and suppression, segmentation of the spaces and ventilation. All these activities are destined to prevent fire spreading in the building. However, there are rare cases when fire overcome these controls and is threatening the occupants and the building. In such cases the management of the emergency scene is delegated to the Incident Commanders (IC). Old buildings were designed with currently obsolete fire engineering approaches. In these buildings fires occur more frequently and generate much more losses. Currently, the forecasting of fire dynamics is bound to the designing of the buildings and not really to the management of the emergency scene by the commanders. This is the result of the complexity of the calculation, which forces the long computation times and the uncertainty of the input parameters for the fire modelling. The geometry of the room and the heat release rate (HRR) are the key input parameters for the successful simulation in the fuel controlled regime. The new achievements in the computer aided design (Building Information Management $\mathrm{BIM}^{1}$ ) allow to hope that in the near future the layouts of the insides of the buildings will be more available, as opposed to the availability of the HRR, unfortunately. The HRR is strongly dependent on the type, amount and distribution of combustible

${ }^{1}$ http://en.wikipedia.org/wiki/Building_information_modeling material inside the room, which is generally difficult to predict or evaluate. Therefore, a set of researches has been conducted which were focused on the evaluation of HRR based on the occurring fire [3], [4], [5]. Each of the approaches in the domain were grouped under one umbrella called inverse fire modelling. However, the approaches are strongly dependent on high sensor density in the compartment, which makes them infeasible during the F\&R actions, taking into account the poor sensors infrastructure in buildings today.

In the previous works on evaluating of the HRR, the authors focused on creating the physical model of the phenomenon. In these models all the parameters represent the physical features. Moreover, their goal was to obtain the strict values of the HRR. These approach is not feasible in the case of F\&R action, where the IC is interested in the approximation of HRR only. During the F\&R action the evaluation of the HRR with accuracy of $\pm 100 \mathrm{~kW}$ is good enough to estimate the type of the fire (furniture, TV, curtains) and its future course of action. Therefore in the case of supporting the IC during the action some conditions can be relaxed.

In this paper we address the problem of inverse fire modelling. We analyze the possibilities of utilizing the inverse fire modeling approach in order to facilitate the management of the emergency scene by the IC. We present a new approach to inverse fire modeling which can be practically used on the emergency scene. The rest of the paper is structured as follows. Section II contains description of our approach and experiments conducted to validate our model. Section III introduces the results obtained in our experiments. In the Section IV we discuss the result obtained and the perspectives of future work for model improvements.

\section{METHOD}

Our method is based on the simple intuition that during the compartment fire, the unique - for a given type of fire imprint of temperature on the ceiling is created. The imprint depends on a set of physical parameters such as HRR, the type of the materials the ceiling is produced from, the geometry of the compartment and others. We also assumed that if we relax 
the conditions for the approximation of HRR purposes then the category of similar compartment will be created allowing for the reduction of invariants considered. It opens the way for running a set of simulations for each of the categories and store them in the database. Then, by comparing the imprint of the actual fire against the completed simulations in the database we can evaluate the HRR and the fire dynamics.

We also assumed that for the approximation purposes during the F\&R action the physical interpretation of the parameters of our model are not important. It allows us for the usage of the approaches from artificial intelligence domain. According to these approaches the feature extraction and their impact on the decision class (HRR value in our case) is obtained automatically during the training process. There is only one demand: large enough amount of training data. This condition is however, easy to meet in our case, because we are able to massively generate the simulations.

In our analysis we focused mainly on the measurement of the temperature distribution on the structural elements of the compartment using IR camera. This method allows to obtain large number of individual measurement points. Number of points and quality of the obtained measurements depends on the accuracy of measuring device. The long-wave IR cameras have become increasingly common in the F\&R actions [6], [7]. In many countries almost all fire departments have at least one IR camera. It helps rescuers to navigate through smoke filled compartments and also speeds up the localization of the fire source. Except from the camera, the rescuers could be equipped with the module for analyzing the temperature distribution on structural elements. This analysis could dynamically estimate many fire parameters such as the HRR, smoke layer temperature, fire area or critical conditions e.g. flash over (combined with information from BIM).

In most cases the thermal response depends on the building materials from which the compartment was made. Building obstructions are mainly heated by radiative and convective heat transfer from fire and conductivity. For large spaces, the uniform heating of walls and ceiling may prove to be a very long process. However, the ceiling above the fire source is always heated identically and comparatively fast. This approach can be universal for all compartments sizes. However, there are many factors that can affect the final imprint of temperatures. Ceiling temperature distribution depends on many variables i.e. HRR, combusted material, fire area, base of the fire, height of the compartment, type of building materials and many others but final imprint is related to the amount of heat released from fire which means that there is a physical dependence of these two parameters.

\section{A. Preliminary experiments}

We conducted the preliminary experiment in order to recognize, whether there are possibilities of using the machine learning algorithms to resolve the problem. We generated, for this purpose, a representative numer of simulations of various fire scenarios in a single compartment. We ran all simulations on the Fire Dynamics Simulator (FDS) [8] software version
6.0.1. FDS is a computational fluid dynamics model of firedriven fluid flow, with an emphasis on smoke and the heat transport from fires. Launching the FDS simulation requires a large number of input parameters. The parameters affect the simulation results. In order to simplify the conditions of preliminary experiment we divided the input parameters into invariants and variants. The setup of invariant parameters is as follows:

- the properties of the building materials,

- height of the room $-2.6 \mathrm{~m}$,

- base of the fire $-0.0 \mathrm{~m}$,

- room dimensions $-5.0 \times 3.0 \mathrm{~m}$,

- burning material - ethyl alcohol,

- FDS model settings except radiation model,

- cell size $-0.2 \mathrm{~m}$,

- fire always is located at least $1.0 \mathrm{~m}$ away from the building walls,

- ventilation hole - entrance door with $1.0 \mathrm{~m}$ width and $2.0 \mathrm{~m}$ height,

- simulation time $-600 \mathrm{~s}$.

The properties of the building materials are crucial in this analysis. Depending on physical properties the different rate of heat loss by the hot gases to compartment boundaries is transfered. We considered the room made of fire resistant calcium silicate boards (both the walls and the ceiling). The assumed physical properties are presented in the Table I.

TABLE I: The physical properties of calcium silicate boards

\begin{tabular}{l|l}
\hline \hline Parameter & Value \\
\hline Density & $480 \mathrm{~kg} / \mathrm{m}^{3}$ \\
Thermal conductivity & $0.09 \mathrm{~W} /(\mathrm{m} \times \mathrm{K})$ \\
Specific heat at $293 \mathrm{~K}$ & $1.074 \mathrm{~kJ} /(\mathrm{kg} \times \mathrm{K})$ \\
Specific heat at $473 \mathrm{~K}$ & $1.000 \mathrm{~kJ} /(\mathrm{kg} \times \mathrm{K})$ \\
Emissivity & 0.9 \\
\hline \hline
\end{tabular}

The parameter specific heat was introduced in order to define the boundary conditions. This parameter is a linear function of the temperature and is defined by two points: $y 1=1.074$ at $293 \mathrm{~K}$ and $y 2=1.0$ at $473 \mathrm{~K}$ (physical parameters of the boards). We also assumed that the height from the fuel source to the ceiling is constant (fire basis) for all the experiments. This parameter as well as the height of the compartment may strongly influence the temperature distribution on the ceiling. The higher is the fire basis the higher temperatures may be reached by ceiling jet. It mainly depends on the plume mass flow above the flames [1]. Moreover, the position of the fire in the room affects these temperatures. If the burning fuel is located close to the walls the cool air is entrained into the plume only from one or two directions. This causes higher temperatures and higher flames from the same fire [9].

We used ethyl alcohol as a burning material. It is commonly used in the real experiments because of its well known physical and chemical properties. It allows to precisely estimate the 
HRR using the following function [10]:

$$
H=345+1139 A-1108 A^{2}+320 A^{3}
$$

where $\mathrm{H}$ is $\mathrm{HRR}$ and $\mathrm{A}$ is the area of the fire.

As variant parameters we assumed the total HRR, the area of the fire and its location in the compartment. We considered steady-state fires with constant HRR which was changing in the range of 50 to $1,000 \mathrm{~kW}$ every $50 \mathrm{~kW}$ for all fire areas and locations. The total number of combinations were 600 .

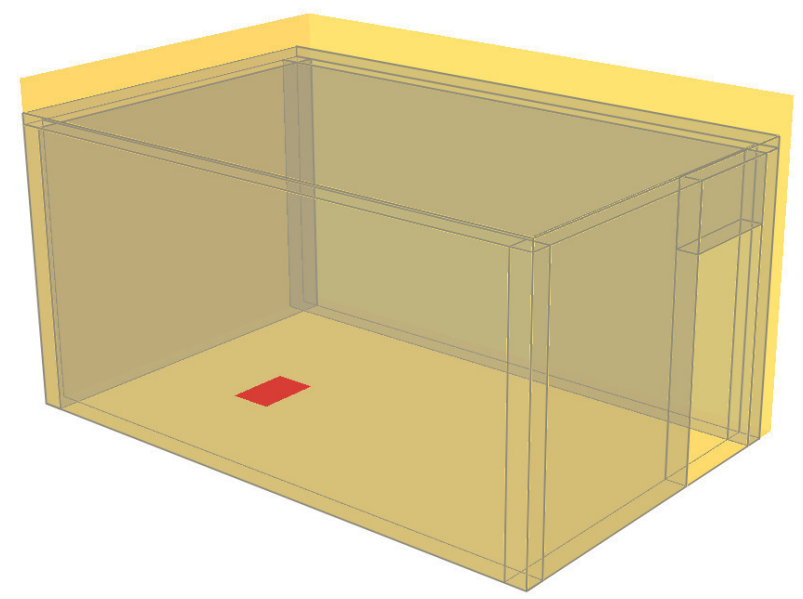

Fig. 1: The compartment view used in the CFD simulations.

Next step of our analysis was the extraction of the data from the simulations. The results e.g., the wall temperature was collected for each cell on all boundaries in the compartment. We recorded the values every 5 seconds. The binary output from the FDS was converted to comma separated values file (CSV) with the fds2ascii tool. All cells from the ceiling were inserted to monetDB ${ }^{2}$ database. Each record contains the information about temperature, location ( $x, y$ coordinate) of the cell ( $\mathrm{z}$ axis fixed) and other data needed to distinguish the fire scenario i.e. HRR, fire area and position. From the total ceiling area only $1 \mathrm{~m}^{2}$ was considered for the analysis this area is chosen by spotting the hottest cell and centering the one square metre around it.

The data from the database was used as a training set for classifiers. We constructed our information system by defining the object as an avaraged values of attributes within defined (30 s) time window of the given fire test. The object in our information system was represented by following attributes: time from ignition, maximum temperature, average temperature form selected area and standard deviation. As a decision class the HRR was used. We used in our experiment the total number of objects (samples) equal 36,600.

Due to the large amount, the data were first discretized. The original values of HRR were in range from 50 to $1,000 \mathrm{~kW}$ with $50 \mathrm{~kW}$ growing step. We conducted two tests with HRR discretized into 20 and 10 equal-width sets. The time of simulation was in the range from 0 to 600 second. We

\footnotetext{
${ }^{2}$ http://www.monetdb.com/Home
}

discretized this parameter into 20 equal-width sets in both cases.

Then we used the orange-canvas ${ }^{3}$ software to run the classifications. We used the 5-folds cross-validation technique for estimating the performance of predictive models. In 5fold cross-validation, the simulations data were randomly partitioned into five equal size subsamples. Out of the five subsamples, a single subsample was retained as the validation data for testing the classifiers, and the remaining four subsamples were used as training data. The cross-validation process was then repeated five times, with each of the five subsamples used exactly once as the validation data. We averaged the five results from the folds to produce a single estimation. The advantage of this method over repeated random sub-sampling is that all observations are used for both training and validation, and each observation is used for validation exactly once.

We used in our experiments two classification algorithms, i.e., Classification Trees [11], [12] and Support Vectors Machine (SVM) [13]. We decided to use these two algoritms because they are based on two different foundations. Classification Trees represents the algoritms with descriptive method of classification. The model created as a result of training process is interpretable and understandable by the fire safety domain experts - the trees with decision rules. The selected algorithm allowed us for better controling the experiments and keeping the physical interpretation of the obtained results. SVM represents respectively the algoritms with procedural method of classification. The model defined within trainig process is not interpretable by domain experts. However, we chose this algorithm for the comparision with Classification Trees and because of good performance in similar problems [14].

The goal of the classifiers was to predict the value of HRR from testing subsample. We used the Balanced Classification Accuracy $(\mathrm{BCA})^{4}$ measure for measuring the performance of the classifiers. At the current state of our researches, the conducted experiments were aimed at checking the possibility of using proposed method. Our intention was not to tune up classifier settings for better performace rather we used default options. We used the following settings of classifiers: SVM type: C-SVM (cost $=1$, complexity bound $=0.50)$, SVM Kernel: $\mathrm{RBF}^{5}$, SVM Numerical tolerance $=0.0010$. Classification Tree attribute selection criterion: information gain, Classification Tree Pre-Running: min. instances in leaves $=2$, pruning with $\mathrm{m}$-estimate $\mathrm{m}=2$.

\section{B. Real experiments}

In order to check whether the method could be applied in the real situation, when the distribution of the temperature on the cell is obtained by the physical equipment, we conducted a set of real experiments. Three surveys of three different HRRs were made within the compartment with dimensions $5.25 \mathrm{~m}$ length, $2.54 \mathrm{~m}$ width and $2.55 \mathrm{~m}$ height. The whole building was made of prefabricated concrete slabs, however

\footnotetext{
${ }^{3}$ http://orange.biolab.si/

${ }^{4} \mathrm{http}$ ://en.wikipedia.org/wiki/Accuracy_and_precision

${ }^{5}$ http://en.wikipedia.org/wiki/Radial_basis_function_kernel
} 
the analyzed compartment was protected by non-combustible low density calcium silicate boards. Fire resistant boards were mounted on steel construction. The compartment had three exits with dimensions $2.1 \times 1.0 \mathrm{~m}$ each. Two of them were closed and the third one, the exit from the building was opened.

The fire source was placed in the compartment at least $1 \mathrm{~m}$ from the walls. $90 \%$ methylated spirits was used as a burning material. Alcohol was burned in steel fire trays based on ISO international paper sizes i.e. A3 and A2, located directly on the floor. The total HRR was estimated according to Australian Standard [10] i.e. $60 \mathrm{~kW} 140 \mathrm{~kW}$ and $200 \mathrm{~kW}$ for A3, A2 and A 3 + A2 fire trays, respectively. Tests were planned to reach steady-state conditions with constant HRR. During the tests the following parameters were recorded:

- imprint of the temperature on the ceiling captured by IR camera,

- temperature distribution in the compartment measured by thermocouple trees,

- video image.

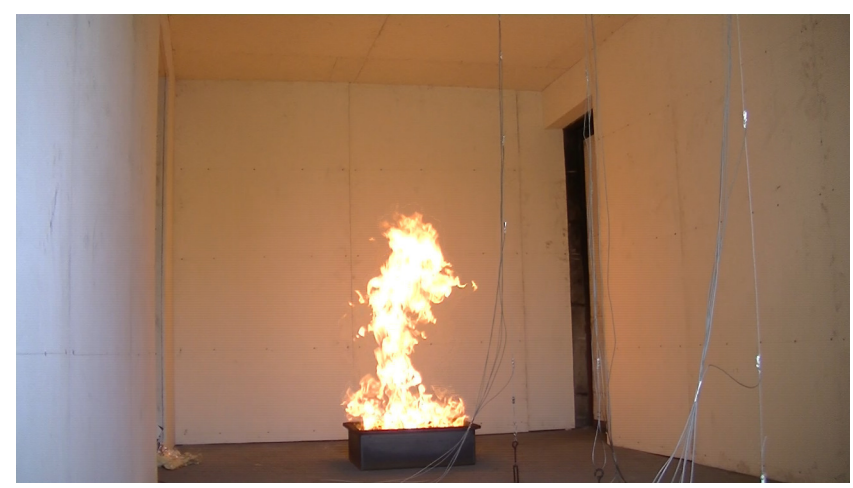

Fig. 2: Experimental setup with A2 fire tray.

Temperature distribution on the ceiling was captured by long-wavelength IR camera - wavelength $8-14 \mu \mathrm{m}$. Resolution of the camera was $640 \times 480$ pixels. The distance from the measured area to the camera was approximately $2.7 \mathrm{~m}$. The pictures were captured every 1 second. We also measured the temperature distribution in compartment using three thermocouple trees which held 6 (Type-K) thermocouples each. These trees were spread in compartment with distance $1 \mathrm{~m}$, $2 \mathrm{~m}, 3 \mathrm{~m}$ from center of the fire source. The values from the thermocouples were logged at frequency of $1 \mathrm{~Hz}$. One video camera was used to monitor the fire growth.

After the fire tests we processed 60 pictures from IR camera. We selected pictures form all tests with a $30 \mathrm{~s}$ step. Next we extracted the attributes needed for the predictions methods i.e. maximum temperature, average temperature and standard deviation from $1 \mathrm{~m}^{2}$. Finally we used already trained classifiers from preliminary experiments to match most suitable decision class.

\section{Towards the Generalization of the Model}

The goal of the experiments described above was a general validation of our approach. It allows for proving that the concepts are reasonable and for evaluating the results. However, the method, even if it obtained quality is satisfying, is not quite practical so far. To make the approach usable, the generation of massive number of simulations for every particular compartment is required. Moreover, taking into account that for a given compartment different conditions of the ventilation could appear (windows and doors could be opened or closed) the generation of the simulation scenarios requires some sampling method such as Monte Carlo. Therefore our next experiments were concentrated on the generic features of the compartments. The extracted features allow to create groups of similar compartments - from the fire scenario point of view. Such grouping, thus allows for relaxing the conditions of generation of the simulations for every particular compartment.

Similarly to preliminary experiments we set a couple of invariant parameters. Most of them were the same except from the following:

- building materials properties,

- height of the room $-2.8 \mathrm{~m}$,

- simulation time $-1,200 \mathrm{~s}$.

This time the test was performed in the room made of gypsum boards. It is a more common material for typical offices or flats. Moreover, we changed the height of the compartment. The previous one was matched to the test room where the real experiments were made.

TABLE II: Variant parameters assumed in simulations.

\begin{tabular}{l|l}
\hline \hline Parameter & $\begin{array}{l}\text { Number } \\
\text { of combination }\end{array}$ \\
\hline Room dimensions & 14 \\
Number of the fire location & 140 \\
Area of the fire & 10 \\
Total heat release rate & 10 \\
\hline Total & 14,000 \\
\hline \hline
\end{tabular}

The assumed variant parameters were presented in Table II. Dimensions of the room were changing from $3 \times 3 \mathrm{~m}$ to $7 \times 7 \mathrm{~m}$ which is corresponding to the real conditions in a dwelling or an office. The increment step was set to $1 \mathrm{~m}$ in both directions except from the repeated dimensions. Also the location of the fire was moving by $1 \mathrm{~m}$, however, the minimal distance from obstructions was set to $1 \mathrm{~m}$. We assumed ten combinations of fire area and total heat release rate i.e. from $0.4 \times 0.4 \mathrm{~m}^{2}$ to $1.0 \times 1.0 \mathrm{~m}^{2}$ and from $100 \mathrm{~kW}$ to $1,000 \mathrm{~kW}$, respectively. The total numer of combinations was 14,000 .

Due to the large number of generated input files the coarse grid size of $20 \mathrm{~cm}$ was chosen. According to previous works [4] and results from preliminary experiments we decided to study the case with the mentioned size of the grid. 


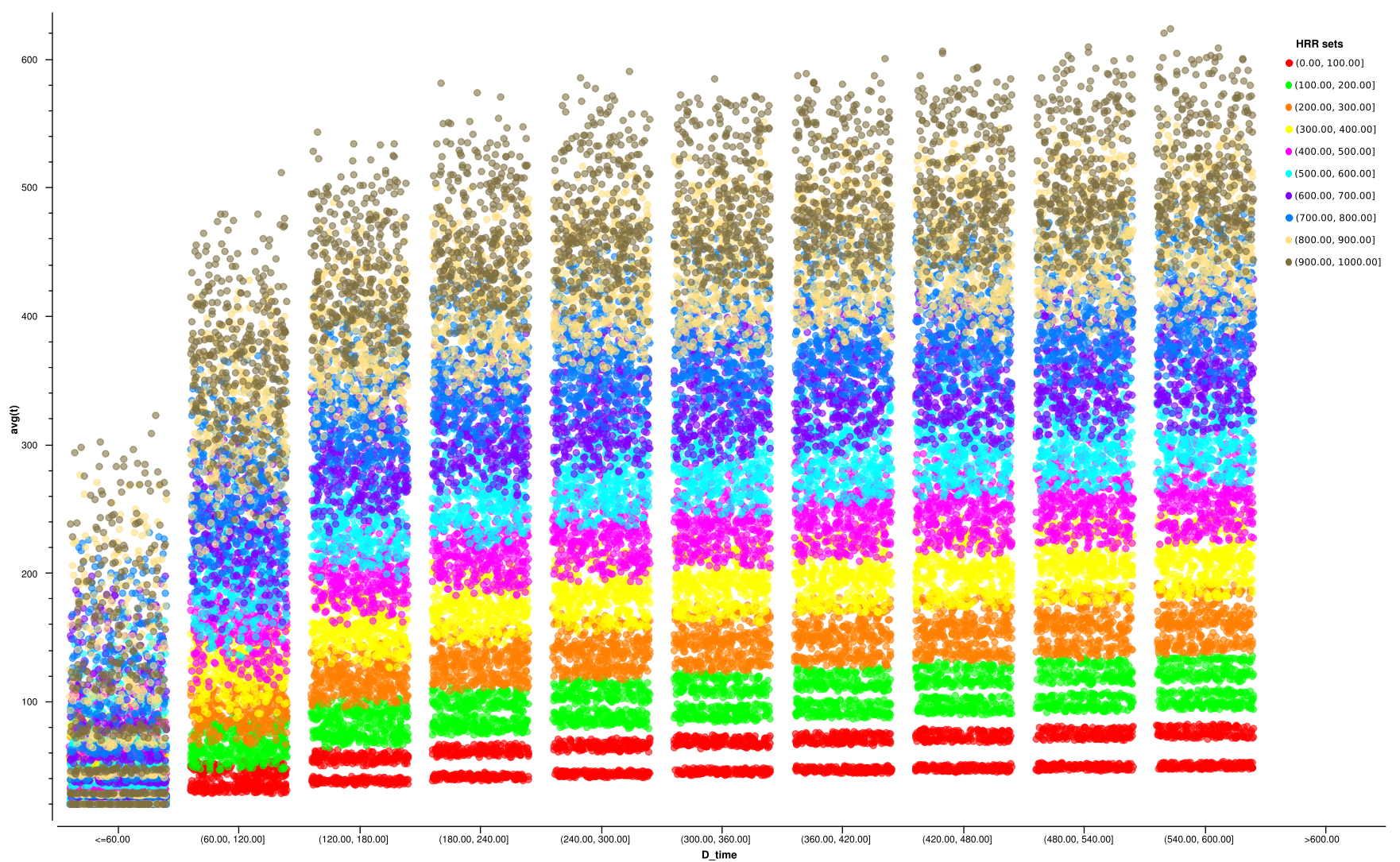

Fig. 3: Temperature distribution on the ceiling in the simulation. Legend: X-axis - discretized time, Y-axis - average temperature.

We chose a similar methodology as in the preliminary experiments for preparing and processing the data for classification methods. We decided upon 10 discrete equal-width sets of the HRR.

\section{RESULTS}

The results were collected in each step of the experiments. Firstly we tested the classification accuracy for preliminary experiments. Then we compared the results from the classifiers against the real experiments. Finally we measured the classification accuracy for generalized model. In all steps we considered BCA for Classification Tree and Support Vector Machine (SVM) classifiers. We also provide the confusion matrix $^{6}$ as the measure of classifiers' performance.

\section{A. Preliminary experiments}

The preliminary experiments aimed at verifying whether the machine learning approach is appropriate for characterizing the HRR from the temperature imprint on the ceiling. We prepared 600 simulations for a single room with varying fire area, fire localization and Heat Release Rate Per Unit Area (HRRPUA). For all the simulations we registered the time, the average and maximum temperatures and the standard deviation of the temperatures in the measured (1 square metre) area. In total there were 36,600 records registered.

\footnotetext{
${ }^{6}$ http://en.wikipedia.org/wiki/Confusion_matrix
}

The Figure 3 illustrates the dependence of the HRR on the average temperature and time. In this case the HRR were discretized into 10 sets. Each set is presented in a distinct color. The figure shows that the sets of the HRRs are quite separable, especially for the lower HRR. For the higher HRR the classification is less certain. However, the additional attributes - maximum temperature and the standard deviation of the temperatures improve the classification of the HRR.

In the Table III we collected the results of the classification accuracy. It shows the results of the test learners for all the classifiers used. We distinguish two groups of the results dependent on the HRR discretization. First group assumes discretization into 20 sets (every $50 \mathrm{~kW}$ ) and the second into 10 sets (every $100 \mathrm{~kW}$ ).

TABLE III: The test learners obtained by the classifiers in the preliminary experiments.

\begin{tabular}{l|cccc}
\hline \hline Test learners & BCA & BCA & AUC & AUC \\
(HRR step) & $50 \mathrm{~kW}$ & $100 \mathrm{~kW}$ & $50 \mathrm{~kW}$ & $100 \mathrm{~kW}$ \\
\hline Classification Tree & 0.6292 & 0.7713 & 0.9745 & 0.9701 \\
SVM & 0.3711 & 0.6727 & 0.9619 & 0.9781 \\
\hline \hline
\end{tabular}

In the Table IV and V we present the confusion matrix for the Classification Tree. The results are for best and worst HRR 


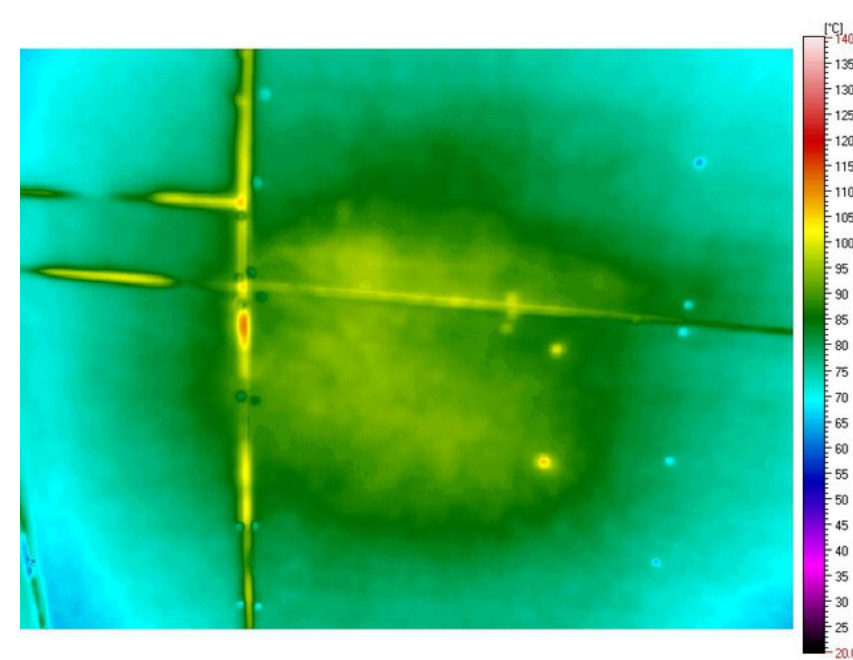

(a) $\mathrm{t}=240$ seconds

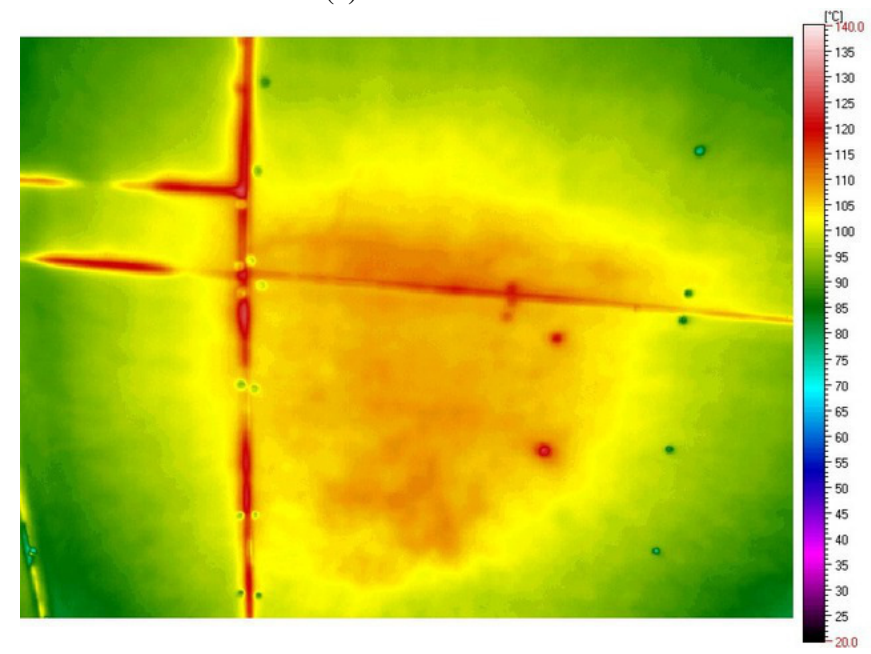

(c) $\mathrm{t}=480$ seconds

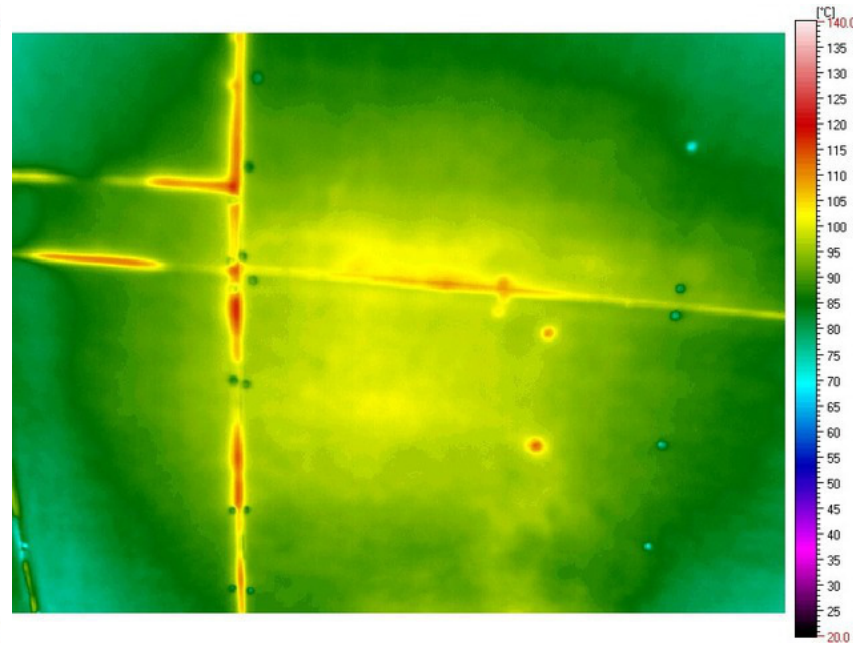

(b) $\mathrm{t}=360$ seconds

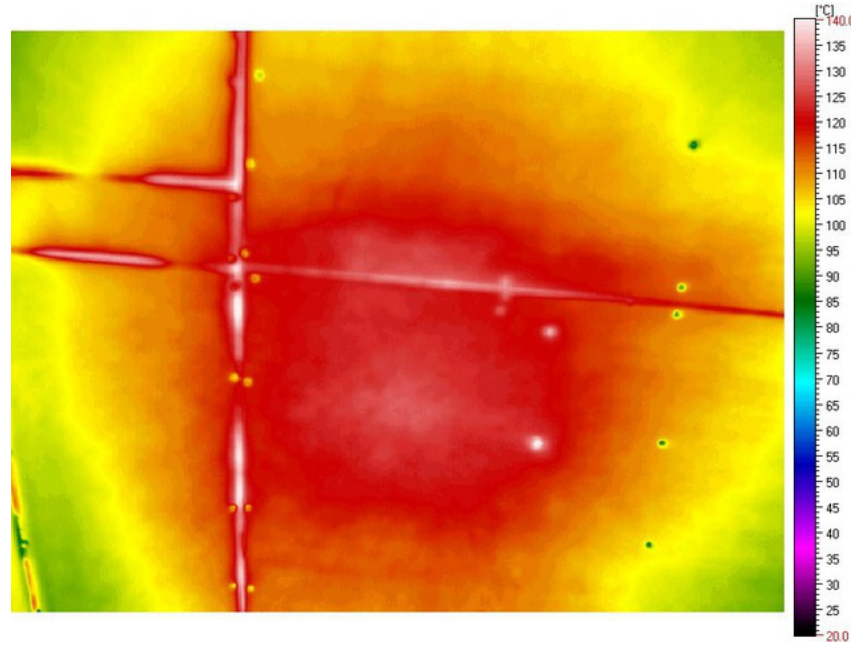

(d) $t=600$ seconds

Fig. 4: Temperature distribution on the ceiling.

classification classes for 20 and 10 equal-width sets (50 and $100 \mathrm{~kW}$ HRR step). It can be observed that the accuracy of the classifier is high because of the low number of false negatives and because these false negatives were assigned to the nearby sets.

\section{B. Real experiments}

The purpose of the study was to validate the classification methods against the results of the full scale experiments. During the real experiments we captured the imprint of the temperature on the compartment ceiling using IR camera. The pictures were captured every second. However, only sixty pictures were used to extract the average and maximum temperatures and the standard deviations of the temperatures. Figure 4 presents the imprints of the ceiling temperature for burning A2 fire tray.

Afterwards we used the prediction module in orange-canvas to verify the classifiers accuracy. In Table VI we present the performance of the classifiers for HRR discretized into 20 and 10 equal-width sets.

TABLE VI: The performance obtained by the classifiers in the real experiments.

\begin{tabular}{l|cc}
\hline \hline Classifier & BCA & BCA \\
& $50 \mathrm{~kW}$ & $100 \mathrm{~kW}$ \\
\hline Classification Tree & 0.5833 & 0.9167 \\
SVM & 0.6667 & 0.900 \\
\hline \hline
\end{tabular}

\section{Generalized model}

In Table VII we present the performance obtained by the classifiers. The total number of all instances was 293,976. 
TABLE IV: Confusion matrix for Classification Tree (50 kW step).

\begin{tabular}{|c|c|c|c|c|c|c|c|c|}
\hline & $(0.00,50.00]$ & $(100.00,150.00]$ & $(200.00,250.00]$ & âĂę & $(700.00,750.00]$ & $(800.00,850.00]$ & $(900.00,950.00]$ & \\
\hline$(0.00,50.00]$ & 1,781 & 7 & 0 & âĂę & 0 & 0 & 0 & 1,830 \\
\hline$(50.00,100.00]$ & 13 & 15 & 2 & âĂę & 0 & 0 & 0 & 1,830 \\
\hline$(100.00,150.00]$ & 5 & 1705 & 7 & âĂę & 0 & 1 & 0 & 1,830 \\
\hline$(150.00,200.00]$ & 4 & 43 & 57 & âĂę & 1 & 0 & 0 & 1,830 \\
\hline$(200.00,250.00]$ & 1 & 11 & 1578 & âĂę & 3 & 0 & 0 & 1,830 \\
\hline$(250.00,300.00]$ & 0 & 4 & 91 & âĂę & 0 & 0 & 3 & 1,830 \\
\hline$(300.00,350.00]$ & 0 & 7 & 22 & âĂę & 4 & 0 & 1 & 1,830 \\
\hline$(350.00,400.00]$ & 0 & 2 & 16 & âĂę & 7 & 1 & 0 & 1,830 \\
\hline$(400.00,450.00]$ & 0 & 8 & 6 & âĂę & 5 & 3 & 4 & 1,830 \\
\hline$(450.00,500.00]$ & 0 & 3 & 10 & âĂę & 8 & 3 & 9 & 1,830 \\
\hline$(500.00,550.00]$ & 0 & 0 & 6 & âĂę & 13 & 2 & 0 & 1,830 \\
\hline$(550.00,600.00]$ & 0 & 0 & 3 & âĂę & 21 & 8 & 5 & 1,830 \\
\hline$(600.00,650.00]$ & 0 & 1 & 2 & âĂę & 69 & 10 & 5 & 1,830 \\
\hline$(650.00,700.00]$ & 0 & 4 & 0 & âĂę & 381 & 24 & 6 & 1,830 \\
\hline$(700.00,750.00]$ & 0 & 1 & 7 & âĂę & 735 & 72 & 18 & 1,830 \\
\hline$(750.00,800.00]$ & 0 & 0 & 13 & âĂę & 378 & 370 & 21 & 1,830 \\
\hline$(800.00,850.00]$ & 0 & 0 & 2 & âĂę & 89 & 757 & 101 & 1,830 \\
\hline$(850.00,900.00]$ & 0 & 0 & 0 & âĂę & 23 & 379 & 375 & 1,830 \\
\hline$(900.00,950.00]$ & 0 & 0 & 0 & âĂę & 23 & 123 & 661 & 1,830 \\
\hline \multirow[t]{2}{*}{$(950.00,1,000.00]$} & 0 & 0 & 0 & âĄę & 9 & 44 & 453 & 1,830 \\
\hline & 1,804 & 1,811 & 1,822 & âĂę & 1,769 & 1,797 & 1,662 & 36,600 \\
\hline
\end{tabular}

TABLE V: Confusion matrix for Classification Tree (100 kW step).

\begin{tabular}{|c|c|c|c|c|c|c|c|c|}
\hline & $(0.00,100.00]$ & $(100.00,200.00]$ & $(200.00,300.00]$ & âĂę & $(700.00,800.00]$ & $(800.00,900.00]$ & $(900.00,1,000.00]$ & \\
\hline$(0.00,100.00]$ & 3,552 & 41 & 25 & âĂę & 23 & 10 & 0 & 3,660 \\
\hline$(100.00,200.00]$ & 49 & 3,430 & 91 & âĂę & 22 & 16 & 0 & 3,660 \\
\hline$(200.00,300.00]$ & 14 & 101 & 3,248 & âĂę & 35 & 13 & 4 & 3,660 \\
\hline$(300.00,400.00]$ & 5 & 38 & 243 & âĂę & 41 & 21 & 2 & 3,660 \\
\hline$(400.00,500.00]$ & 4 & 27 & 48 & âĂę & 35 & 29 & 18 & 3,660 \\
\hline$(500.00,600.00]$ & 2 & 21 & 32 & âĂę & 80 & 35 & 17 & 3,660 \\
\hline$(600.00,700.00]$ & 0 & 21 & 18 & âĂę & 594 & 55 & 34 & 3,660 \\
\hline$(700.00,800.00]$ & 0 & 17 & 28 & âĂę & 2,285 & 607 & 60 & 3,660 \\
\hline$(800.00,900.00]$ & 0 & 20 & 18 & âĂę & 624 & 2,185 & 696 & 3,660 \\
\hline \multirow[t]{2}{*}{$(900.00,1000.00]$} & 0 & 12 & 25 & âĂę & 113 & 634 & 2,800 & 3,660 \\
\hline & 3,626 & 3,728 & 3,776 & âĂę & 3,852 & 3,605 & 3,631 & 36,600 \\
\hline
\end{tabular}

TABLE VII: The performance obtained by the classifiers in the generalized method.

\begin{tabular}{l|l}
\hline \hline Classifier & BCA \\
\hline Classification Tree & 0.6569 \\
SVM & 0.5436 \\
\hline \hline
\end{tabular}

\section{DISCUSSION}

The presented results prove that there is a potential in the described approach. We reached a high value of BCA (0.77) in the preliminary experiments. No less optimistic are the results of the real experiments with the IR camera. These experiments resulted in BCA equal 0.92 . The separability of the (especially lower) HRR in the analyzed data were observed. In order to improve the performance in higher HRR, the observed area of the ceiling should be extended by lower focal length of the objective of IR camera. There is a room for other more sophisticated statistics and operations on the features from the pictures, e.g. calculating the parameters of spatial distribution of temperature instead of the mean etc.

The main goal of the research was to asses whether the proposed approach may be used on the fire ground. The results from the real experiments showed that this method may be used to characterizing HRR. The BCA ratio for full scale experiments could be higher, however there were problems with selecting correct values of the attributes from IR camera pictures. In many cases products of combustion i.e. water vapour and aerosols radiation veil created imprint on the ceiling. In order to overcome these shortcomings we consider processing of the images to get the lowest temperatures from the analyzed set of frames. For this purpose we will record the sequence of frames and then use the fluctuating phenomenon of flame and gases to obtain the points of interest. Obviously we may face further problems with more dense smoke where soot yield is higher and the combustion reaction is incomplete. However, we also consider to use mid-wave IR camera to find 
regions in infrared spectrum where these particles emit less electromagnetic wave.

The proposed method has a number of general assumptions. Most of them determined the final results of generated simulations and the type of the temperature imprint. In our opinion most decisive parameters are the fire base, fire localization and physical properties of the building materials. In all tests we located the fire on the floor and at least $1 \mathrm{~m}$ from the walls. Both of these parameters may strongly affect the amount of the incoming air to the convection column which influences the temperatures reached in combustion process. Moreover various building materials show different speed of conduction process. Even though the density, specific heat, thermal conductivity or emissivity may affect the results, we hope to distinguish the specific groups of materials in common buildings.

Further problems may be encountered if the fire varies in its area and its total HRR. In our analyses we considered the fire with fixed area and constant HRRPUA. Both factors have crucial influence on temperature imprint on the ceiling, however to check usefulness of machine learning method these assumptions were made.

Another problem may arise when few fire sources occur in considered compartment. Our method can deal with only one source of fire. The main goal is to localize the warmest area on the ceiling and then extract the needed attributes. This assumption may be inappropriate if there are multiple sources of fire.

At this stage of the research we are also uncertain about the number of exemplars of compartment which should be used in order to evaluate most of the fire scenarios. If we were to consider each small difference in geometry, ventilation conditions and other parameters then we would be forced to generate and store an enormous amount of simulations which is impractical. However, we choose the sensible experiments scenarios and thus limit their number.

One of the parameters which was used as a descriptor of the current condition of the fire (except from the temperature) was time from the beginning of the fire. This requires that the IC provides the accurate (within 1 minute) time of the ignition. Less experienced ICs may be not able to evaluate the time so accurately, which makes the whole approach fail. This will not be an issue for the fires detected by fire detecting systems with the time log.

In our further works we will focus on finding principal factors determining the results of the simulations. For this purpose we will consider the application of Principal Component Analysis (PCA) ${ }^{7}$, Non-negative matrix factorization $(\mathrm{NMF})^{8}$ methods or rough set approach ${ }^{9}$. All these approaches will be used in order to determine the most important features which affect the temperature imprint on the ceiling. This allows us for the more comprehensive addressing the problem of reduction of the number of generated simulations for a specific

\footnotetext{
${ }^{7}$ http://en.wikipedia.org/wiki/Principal_component_analysis

${ }^{8} \mathrm{http} / / /$ en.wikipedia.org/wiki/Non-negative_matrix_factorization

${ }^{9}$ http://en.wikipedia.org/wiki/Rough_set
}

compartment. After that we will try to generate a new set of the simulations including research results and create new classifiers for this set. Even later we will prepare the full-scale experiments with various ceiling materials and various HRR.

\section{ACKNOWLEDGMENTS}

The research was supported by the Polish National Centre for Research and Development (NCBiR) - Grant No. O $\mathrm{ROB} / 0010 / 03 / 001$ in the frame of Defence and Security Programmes and Projects: "Modern engineering tools for decision support for commanders of the State Fire Service of Poland during Fire\&Rescue operations in the buildings".

\section{REFERENCES}

[1] D. Drysdale, An introduction to fire dynamics. John Wiley \& Sons, 2011

[2] B. Karlsson and J. Quintiere, Enclosure fire dynamics. CRC press, 2002

[3] W. Jahn, G. Rein, and J. L. Torero, "Forecasting fire growth using an inverse zone modelling approach," Fire Safety Journal, vol. 46, no. 3 , pp. 81-88, 2011.

[4] W. Jahn, J. L. Torero, and G. Rein, "Forecasting fire dynamics using inverse computational fluid dynamics and tangent linearisation," Advances in Engineering Software, vol. 47, no. 1, pp. 114-126, 2012.

[5] K. J. Overholt and O. A. Ezekoye, "Characterizing Heat Release Rates Using an Inverse Fire Modeling Technique," Fire Technology, vol. 48, no. 4, pp. 893-909, 2012

[6] J. Martinez-de Dios, B. C. Arrue, A. Ollero, L. Merino, and F. GómezRodríguez, "Computer vision techniques for forest fire perception," Image and vision computing, vol. 26, no. 4, pp. 550-562, 2008.

[7] J. San-Miguel-Ayanz and N. Ravail, "Active fire detection for fire emergency management: Potential and limitations for the operational use of remote sensing," Natural Hazards, vol. 35, no. 3, pp. 361-376, 2005.

[8] R. M. J. F. C. W. K. O. K. McGrattan, S. Hostikka, Fire Dynamics Simulator, User's Guide, 2013.

[9] B. Y. Lattimer, "Heat Fluxes from Fires to Surfaces," in The SFPE Handbook of Fire Protection Engineering, $3^{\text {rd }}$ Edition, 2002, pp. 2 269 - 2-296.

[10] Australian Standard AS 4391-1999, Smoke managment systems - Hot smoke test, 1999.

[11] X. Wu, V. Kumar, J. R. Quinlan, J. Ghosh, Q. Yang, H. Motoda, G. J. McLachlan, A. Ng, B. Liu, S. Y. Philip et al., "Top 10 algorithms in data mining," Knowledge and Information Systems, vol. 14, no. 1, pp. $1-37,2008$.

[12] I. H. Witten and E. Frank, Data Mining: Practical machine learning tools and techniques. Morgan Kaufmann, 2005.

[13] M. A. Hearst, S. Dumais, E. Osman, J. Platt, and B. Scholkopf, "Support vector machines," Intelligent Systems and their Applications, IEEE vol. 13 , no. 4, pp. 18-28, 1998.

[14] S. Wang, W. Zhu, and Z.-P. Liang, "Shape deformation: Svm regression and application to medical image segmentation," in Computer Vision, 2001. ICCV 2001. Proceedings. Eighth IEEE International Conference on, vol. 2. IEEE, 2001, pp. 209-216. 